\title{
Mesoscopic Fano effect in a spin splitter with a side-coupled quantum dot
}

\author{
V. Moldoveanu ${ }^{\mathrm{a}}$, M. Ţolea ${ }^{\mathrm{a}}, \mathrm{B}$. Tanatar $^{\mathrm{b}, *}$ \\ a National Institute of Materials Physics, P.O. Box MG-7, Bucharest-Magurele, Romania \\ b Department of Physics, Bilkent University, Bilkent, 06800 Ankara, Turkey
}

\section{A R T I C L E I N F O}

\section{Article history:}

Received 12 April 2011

Accepted 11 September 2011

Available online 10 February 2012

Communicated by V.M. Agranovich

\section{Keywords:}

Rashba spin-orbit coupling

Fano effect

Mesoscopic rings

\begin{abstract}
A B S T R A C T
We investigate the interplay between the spin interference and the Fano effect in a three-lead mesoscopic ring with a side-coupled quantum dot (QD). A uniform Rashba spin-orbit coupling and a perpendicular magnetic field are tuned such that the ring operates as a spin splitter in the absence of the QD: one lead is used to inject unpolarized electrons and the remaining (output) leads collect almost polarized spin currents. By applying a gate potential to the quantum dot a pair of spin-split levels sweeps the bias window and leads to Fano interference. The steady-state spin and charge currents in the leads are calculated for a finite bias applied across the ring via the non-equilibrium Green's function formalism. When the QD levels participate to transport we find that the spin currents exhibit peaks and dips whereas the charge currents present Fano lineshapes. The location of the side-coupled quantum dot and the spin splitting of its levels also affect the interference and the output currents. The opposite response of output currents to the variation of the gate potential allows one to use this system as a single parameter current switch. We also analyze the dependence of the splitter efficiency on the spin splitting on the QD.
\end{abstract}

(C) 2012 Elsevier B.V. All rights reserved.

\section{Introduction}

The role of the Rashba spin-orbit (SO) coupling on the transport properties of mesoscopic rings has been the subject of extensive theoretical studies (see e.g. [1-4] and references therein). It is by now well understood that the spin interference effects appearing due to the different precession angles between the left and right branches $[5,6]$ can be used to operate the ring as a spin filter or as a spin splitter [7-12]. The splitter regime of a 1D mesoscopic ring with one input lead and two output leads was introduced by Földi et al. [7]. The main feature of the splitter is that even if the input lead carries unpolarized spins, the spin interference in the ring can be tuned such that one output lead provides 'almost' spinup electrons while the other one 'almost' spin-down electrons.

In our recent work $[11,12]$ on mesoscopic rings we presented a systematic study of both spin-filter and spin-splitter operations for a one-dimensional ring with Rashba SO coupling. We emphasized that under certain values of a perpendicular magnetic field and of the Rashba coupling strength $\alpha_{R}$, the interference between the clockwise and counterclockwise spin waves becomes constructive or destructive and that this fact insures spin filtering. The main step forward we achieved with respect to previous work is the calculation of the spin-polarized currents at finite bias, instead of the energy-dependent transmittances (see e.g. [10]).

\footnotetext{
* Corresponding author.

E-mail address: tanatar@fen.bilkent.edu.tr (B. Tanatar).
}

This Letter aims to extend our study further considering the spin-dependent interference in a more complicated system, namely a Rashba ring with a quantum dot side-coupled to one of its arms. At first glance this setup is very similar to well-known AharonovBohm ( $\mathrm{AB}$ ) interferometer with an embedded quantum dot which was extensively studied both theoretically and experimentally in the context of the mesoscopic Fano effect (see the pioneering experiment of Kobayashi et al. [13] and the reviews [14,15]). The Fano interference originates from the two different contributions to transport: the so-called 'background' signal which is due to the electrons traveling around the ring without tunneling through the dot and the 'resonant' part given by electrons tunneling through the dot at least once before leaving the ring. Let us stress here that the mesoscopic Fano effect in a two-lead quantum ring with a side-coupled dot has been already experimentally observed by Fuhrer et al. [16] in the absence of the spin-orbit coupling.

The effect of the spin-orbit coupling in $A B$ rings with quantum dots was also investigated theoretically but from a different point of view: the dot itself is supposed to support spin-flip processes while the ring has $\alpha_{R}=0$ (see e.g. [17,18]). This problem is of considerable interest as the electrons in the QD interact, a fact which leads to Coulomb blockade and/or Kondo effects [19]. It has to be mentioned as well that most of these studies consider a two-lead geometry and that the spin-splitter problem is not addressed.

Rather than further investigating the above scenario, we shall turn the problem around. Consider a three-lead Rashba ring set in the spin-splitter regime by appropriately adjusting $\alpha_{R}$ and the magnetic flux. The main questions we shall address are the 
following. (1) Could we still control the spin filtering if a quantum dot is side-coupled to the ring? (2) What is the effect of the side-coupled dot on the spin and charge currents? To the best of our knowledge these issues have not been addressed before at the theoretical level, even though the side-coupled QD setup can be realized in experiments without much difficulty, as proved by Fuhrer et al. [16].

We shall use the non-equilibrium Green's function formalism which allows us to analyze the splitter regime in the finite bias case. We do not include the effect of the electron-electron interaction among electrons in the ring. Also we do not consider here the effect of the Coulomb interaction in the dot. This is a reasonable approach as long as the coupling between the dot and the ring is weak and the Kondo effect is therefore absent. Coulomb blockade effects could be instead described within a mean-field model but this will not alter the main findings.

The rest of this Letter is organized as follows. In Section 2 we briefly review the model Hamiltonian and the equations leading to the calculation of spin and charge currents. Section 3 contains the numerical results and their discussion while Section 4 is left to conclusions.

\section{Formalism}

The quasi-one-dimensional noninteracting ring is modeled by a 1D lattice containing $N$ sites, each site $p$ corresponding to an angle $\varphi_{p}=2 \pi(p-1) / N$, with $p=1, \ldots, N$. The ring's Hamiltonian $H^{R}$ was explicitly given in Refs. $[11,12]$ in the basis $\{p, \sigma\}$ where $\sigma=\uparrow, \downarrow$ are spin orientations with respect to the $z$-axis. It is derived by a standard discretization of the Hamiltonian presented by Meijer et al. [20] (see also a detailed recent derivation [21]).

For simplicity we adopt a single-site model for the side-coupled dot; adding more structure is clearly possible in our approach but will not alter the main findings on the Fano interference (the system is schematically shown in Fig. 1). This assumption means that the contribution of the side-coupled quantum dot is mostly given by two spin-split levels $\epsilon_{\lambda}$, where $\lambda=\Uparrow, \Downarrow$. More precisely, $\epsilon_{\Uparrow, \Downarrow}=\epsilon_{0}+V_{g} \pm \frac{\Delta}{2}$ where $\epsilon_{0}$ is the on-site energy and $V_{g}$ is the gate potential applied on the dot. We use the notation $\Uparrow, \Downarrow$ in order to avoid the confusion with up and down $(\uparrow, \downarrow)$ spins on the leads. The splitting $\Delta$ is considered to be a tunable parameter in the numerical simulations. It can be due to the Zeeman splitting and/or to an additional intradot Rashba coupling. The tunneling amplitude between the QD and the site $p_{0}$ of the ring is denoted by $\tau_{\mathrm{r}}$. The Fano regime implies that $\tau_{\mathrm{r}}$ is much smaller than the hopping between the leads and the ring which shall be denoted by $\tau$. With these notations the total Hamiltonian reads as $\left(t_{L}\right.$ is the hopping energy on the leads):

$H(t)=H_{0}+H_{L}+H_{T}(t)$,

where

$$
\begin{aligned}
H_{0}= & \sum_{p, p^{\prime}=1}^{N} \sum_{\sigma, \sigma^{\prime}=\uparrow, \downarrow} H_{p \sigma, p^{\prime} \sigma^{\prime}}^{R}|p \sigma\rangle\left\langle p^{\prime} \sigma^{\prime}\right| \\
& +\sum_{\lambda=\Uparrow, \Downarrow} \epsilon_{\lambda}|\lambda\rangle\langle\lambda| \\
& +\tau_{\mathrm{r}}\left(\left|p_{0} \uparrow\right\rangle\left\langle\Uparrow|+| p_{0} \downarrow\right\rangle\langle\downarrow|+\text { h.c. }\right), \\
H_{L}= & t_{L} \sum_{\alpha} \sum_{n_{\alpha} \geqslant 0, \sigma}\left(\left|n_{\alpha} \sigma\right\rangle\left\langle n_{\alpha}+1, \sigma\right|+\text { h.c. }\right), \\
H_{T}(t) & =\chi(t) \sum_{\alpha} \sum_{\sigma}\left(V^{\alpha}\left|0_{\alpha} \sigma\right\rangle\left\langle p_{\alpha} \sigma\right|+\text { h.c. }\right) .
\end{aligned}
$$

$H_{0}$ is nothing but the Hamiltonian of the ring with the sidecoupled dot attached at site $p_{0}$. Note that the matrix elements

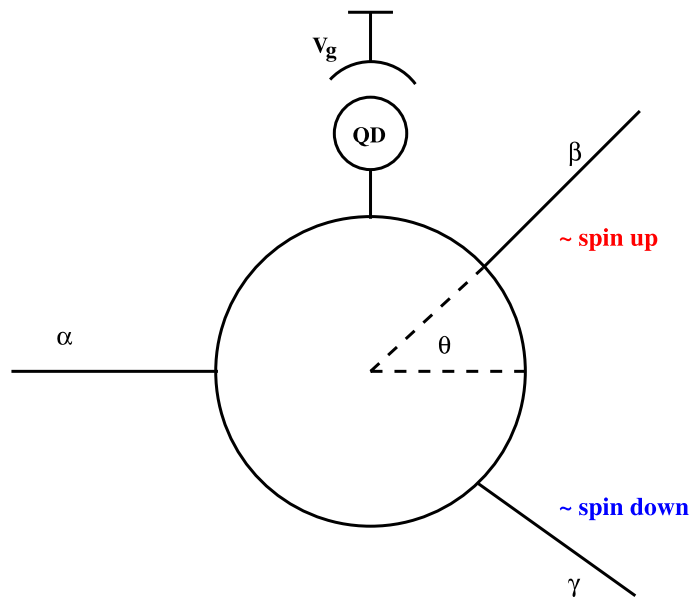

Fig. 1. (Color online.) The sketch of the Rashba splitter with the side-coupled QD. By suitably tuning the magnetic flux and the Rashba spin-orbit coupling the output lead $\beta$ delivers mostly up-spins while the lead $\gamma$ mostly down-spins. The gate potential $V_{g}$ controls the transport through the $\mathrm{QD}$.

$H_{p \sigma, p^{\prime} \sigma^{\prime}}^{R}$ are complicated expressions (see $[11,12]$ ) containing the levels of the bare ring and the eigenfunctions of the discretized ring. The latter are denoted by $\psi_{l s}$ where $s= \pm$ is the spin quantum number in the local spin frame and $l=0, \pm 1, \ldots, \pm(N / 2-$ $1), N / 2$ is the orbital quantum number. $\left|\psi_{l+}\right\rangle$ and $\left|\psi_{l-}\right\rangle$ are given by:

$$
\begin{aligned}
& \left|\psi_{l+}\right\rangle=\left(\begin{array}{c}
\cos \left(\frac{\omega_{l}}{2}\right)\left|\phi_{l}\right\rangle \\
\sin \left(\frac{\omega_{l}}{2}\right)\left|\phi_{l+1}\right\rangle
\end{array}\right), \\
& \left|\psi_{l-}\right\rangle=\left(\begin{array}{c}
-\sin \left(\frac{\omega_{l}}{2}\right)\left|\phi_{l}\right\rangle \\
\cos \left(\frac{\omega_{l}}{2}\right)\left|\phi_{l+1}\right\rangle
\end{array}\right),
\end{aligned}
$$

where the tilt angle $\omega_{l}$ depends on the Rashba coupling (see [1] for its expression in the continuous model - here we use a discretized analogue). $\left|\phi_{l}\right\rangle$ are the eigenfunctions of the discretized ring in the absence of Rashba coupling.

The coupling between the leads and the ring implies a pair of sites $\left(0_{\alpha}, p_{\alpha}\right)$, where $p_{\alpha}$ is the site of the ring where the lead is attached and $0_{\alpha}$ is the nearest site of the lead (the sites of the lead $\alpha$ are denoted by $n_{\alpha}$ ). Note that the coupling between the ring and the dot does not couple opposite spins (e.g. $\uparrow$ and $\Downarrow$ ); this means that no spin-flip processes occur when electrons tunnel in and from the dot. $V_{\alpha}$ is the hopping constant between the dot and the lead $\alpha$. We assume that the spin of the incident electron is not changed at the contacts. The time-dependence in the tunneling Hamiltonian $H_{T}$ is due to a smooth switching function $\chi(t)$ which is needed in order to have a well defined initial state (i.e. the state of the disconnected system). $H_{L}$ is the Hamiltonian of the leads.

The steady-state currents are calculated in a standard way within the non-equilibrium Green's function formalism. In the non-interacting case the key ingredients are the 'effective' advanced and retarded Green's functions:

$G^{R, A}(E)=\left(E-H_{0}-\Sigma^{R, A}\right)^{-1}$,

where as usual $\Sigma^{R, A}$ are leads' self-energies (see Eq. (16) in [11]). Note that both the Green's functions and the self-energies are matrices containing both site indices and spin polarizations (see [11] for further details). The steady-state current entering the lead $\alpha$ reads as:

$$
\begin{aligned}
J_{\alpha}= & \frac{e}{h} \int_{-2 t_{L}}^{2 t_{L}} d E \operatorname{Tr}\left\{\Gamma_{\alpha} G^{R} \Gamma_{\beta} G^{A}\left(f_{\alpha}-f_{\beta}\right)\right. \\
& \left.+\Gamma_{\alpha} G^{R} \Gamma_{\gamma} G^{A}\left(f_{\alpha}-f_{\gamma}\right)\right\} .
\end{aligned}
$$


The currents leaving the ring through the leads $\beta$ and $\gamma$ are defined in a similar way:

$$
\begin{aligned}
J_{\beta}= & \frac{e}{h} \int_{-2 t_{L}}^{2 t_{L}} d E \operatorname{Tr}\left\{\Gamma_{\beta} G^{R} \Gamma_{\alpha} G^{A}\left(f_{\alpha}-f_{\beta}\right)\right. \\
& \left.+\Gamma_{\beta} G^{R} \Gamma_{\gamma} G^{A}\left(f_{\gamma}-f_{\beta}\right)\right\}, \\
J_{\gamma}= & \frac{e}{h} \int_{-2 t_{L}}^{2 t_{L}} d E \operatorname{Tr}\left\{\Gamma_{\gamma} G^{R} \Gamma_{\alpha} G^{A}\left(f_{\alpha}-f_{\gamma}\right)\right. \\
& \left.+\Gamma_{\gamma} G^{R} \Gamma_{\beta} G^{A}\left(f_{\beta}-f_{\gamma}\right)\right\} .
\end{aligned}
$$

The linewidths $\Gamma$ are related to the density of states at the endpoint of the lead $\rho(E)=\sqrt{4 t_{L}^{2}-E^{2}} / \pi \quad(\nu=\alpha, \beta, \gamma)$, i.e. $\Gamma_{p \sigma, p^{\prime} \sigma^{\prime}}^{\nu}(E)=\left(V^{v}\right)^{2} \delta_{p p_{v}} \delta_{p^{\prime} p_{\nu}} \rho(E)$. In the above equations the trace means a sum over both site indices and spin indices. One can then identify the spin currents $J_{\uparrow, \downarrow}^{v}$ in lead $v$. Each lead is characterized by its Fermi function and the bias applied between the two leads is as usual given by the difference between their chemical potentials. Let us stress that one can choose different biases between the input and output leads. However for simplicity we take $\mu_{\beta}=\mu_{\gamma}$ and the bias is given by $V=\mu_{\alpha}-\mu_{\beta}=\mu_{\alpha}-\mu_{\gamma}$. We now introduce the efficiency of the spin splitter [10]:

$E_{\uparrow, \downarrow}=\frac{\left(J_{\beta, \uparrow}-J_{\beta, \downarrow}\right)\left(J_{\gamma, \downarrow}-J_{\gamma, \uparrow}\right)}{\left(J_{\beta, \uparrow}+J_{\beta, \downarrow}\right)\left(J_{\gamma, \downarrow}+J_{\gamma, \uparrow}\right)}$,

which depends both on the magnetic field and on the gate potential $V_{g}$. An ideal splitter implies $J_{\beta, \downarrow}=J_{\gamma, \uparrow}=0$. The opposite situation is characterized by the efficiency $E_{\downarrow, \uparrow}$ which is defined in a similar way, by exchanging $\uparrow$ and $\downarrow$.

\section{Numerical results}

Let us consider a ring of radius $R=80 \mathrm{~nm}$ containing $N=80$ sites and submitted to a constant perpendicular magnetic field $B_{z}$. The hopping energy of the leads is $t_{L}=\hbar^{2} / 2 m^{*} a^{2}$, where $a$ is the discretization constant on the ring ( $a=6.28 \mathrm{~nm}$ in this case). We take equal coupling to the leads $V^{\alpha}=V^{\beta}=V^{\gamma}=\tau=0.5$ and $\tau_{\mathrm{r}}=0.001$. The chemical potentials of the leads are set to $\mu_{\alpha}=11.5 \mathrm{meV}$ and $\mu_{\beta}=\mu_{\gamma}=10.5 \mathrm{meV}$. The two output leads $\beta$ and $\gamma$ are located symmetrically w.r.t. the $x$-axis the corresponding angle being denoted by $\theta$ (see Fig. 1 ). The contacts sites $p_{\beta}=6$ and $p_{\gamma}=76$, such that $\theta_{\beta}=\pi / 8$ and $\theta_{\gamma}=2 \pi-\theta_{\beta}$. Unless otherwise stated, the quantum dot connects to the ring at site no. 21 (i.e. $\theta=\pi / 2$ ). We assume that the splitting $\Delta$ of the QD levels can be freely varied and that it can be even considerably larger than the Rashba and Zeeman splitting of the ring's levels. Experimentally this can be achieved in various ways. First the QD could be submitted to an additional magnetic field $B_{\mathrm{QD}}$, hence the Zeeman splitting $\Delta=g_{\mathrm{QD}} \mu_{B} B_{\mathrm{QD}}$ increases by increasing the magnetic field $B_{\mathrm{QD}}$. Also, a tunable Rashba spin-orbit coupling within the QD itself can lead to a larger spin splitting. For simplicity we shall express $\Delta$ as multiples of the Zeeman splitting $g \mu_{B} B_{z}$ on the ring, i.e. $\Delta=M g \mu_{B} B_{z}$, where $M$ is an integer.

Fig. 2a shows the region of the spectrum of the ring $+Q D$ system around the bias window as a function of $V_{g}$. This is the relevant region for the transport problem at hand since the levels outside the bias window will not contribute significantly to transport. The four 'traces' seen for $V_{g}<7 \mathrm{meV}$ correspond to spin-up and spin-down states propagating clockwise and counterclockwise. Note that the labels 'up' and 'down' refer here to the orientation in the local spin frame $s=+,-$. As expected the gate potential induces a slope on the QD levels as long as the latter are far away
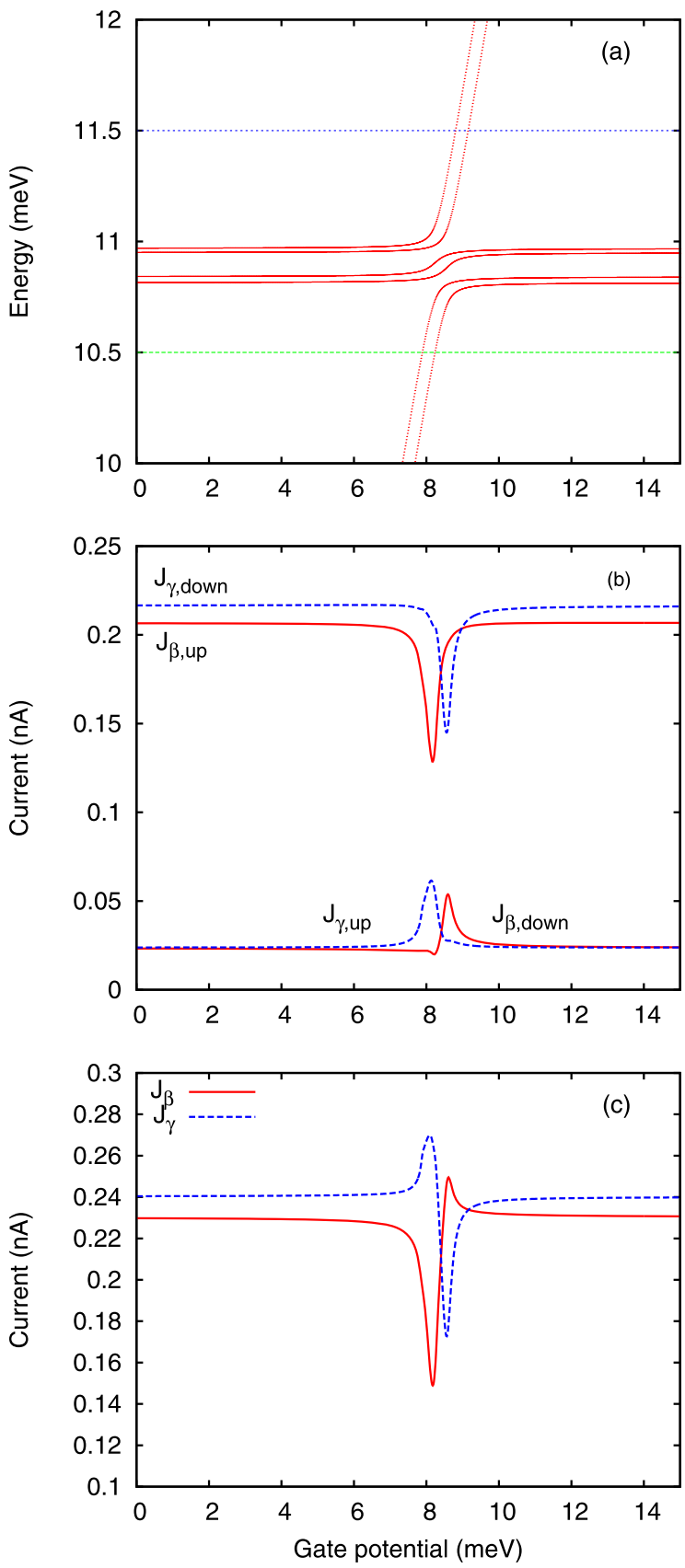

Fig. 2. (Color online.) (a) The spectrum of a Rashba ring of radius $R=80 \mathrm{~nm}$ with a side-coupled dot, as a function of the gate potential $V_{g}$. The vertical lines mark the chemical potentials of the leads (i.e. $\mu_{\alpha}=11.5 \mathrm{meV}$ and $\mu_{\beta}=\mu_{\gamma}=10.5 \mathrm{meV}$ ). The two spin-split levels of the quantum dot enter the bias window as $V_{g}$ increases. The avoided crossings mark the hybridization between the ring's spectrum and the QD levels. (b) The spin currents in the output leads: $J_{\beta, \uparrow, \downarrow}-$ solid line, $J_{\gamma, \uparrow, \downarrow}-$ dashed line. (c) The Fano lineshapes of the charge currents $J_{\beta}$ - solid line, $J_{\gamma}-$ dashed line. The resonances develop as the QD levels sweep the bias window. Other parameters are $\alpha_{R}=0.27 \times 10^{-11} \mathrm{eVm}, \Phi=0.99 \Phi_{0}, \Delta=30, \tau_{r}=0.001$.

from the levels of the bare ring. The ring-dot coupling leads also to a hybridization of the two spectra. As a consequence avoided crossings develop within the bias window in Fig. 2a. The selected spin splitting of the QD levels is much larger than the splitting of the ring levels, for the clarity of the figure. Note that for $V_{g} \sim 8 \mathrm{meV}$ one has six levels within the bias window; the associated states are no longer localized either in the ring or on the dot. This is the regime where the Fano effect and the Rashba interference coexist because on one hand the electrons can tunnel through the dot and 
on the other hand the spin interference between various spinors still takes place.

We select the magnetic flux and the strength of the Rashba coupling $\alpha_{R}$ such that the ring operates as a spin splitter at least when the quantum dot is either absent or the transport through it is forbidden. From our previous study [12] we know that the splitter regime is to be found around integer multiples of $\Phi / \Phi_{0}$, therefore, here we set $\Phi=0.99 \Phi_{0}$. The selected bias window $\left[\mu_{\alpha}, \mu_{\beta}\right]$ covers just one avoided crossing region from the spectrum of the ring (see Fig. 2a).

Fig. 2b displays the spin-up and spin-down currents in the output leads as a function of the gate potential $V_{g}$ applied on the QD. Clearly, as long as the QD is off-resonance (i.e. for $V_{g}<7 \mathrm{meV}$ ) $J_{\beta, \uparrow} \sim J_{\gamma, \uparrow}$ exceed by far $J_{\beta, \downarrow}$ and $J_{\gamma, \uparrow}$.

Moreover, the splitter efficiency is around 65\% (see Fig. 3a). The Fano interference emerges as the spin-up level of the QD approaches and enters the bias window. Fig. $2 \mathrm{~b}$ reveals that at resonance $J_{\beta, \uparrow}$ drops while $J_{\gamma, \uparrow}$ exhibits a moderate peak. The spin-down currents show a similar behavior at a slightly larger $V_{g}$; looking at Fig. 2a we infer that this happens because the second QD level $\epsilon_{\Downarrow}$ enters the bias window later than $\epsilon_{\Uparrow}$.

The fact that the quantum dot participates in transport is also confirmed by the drop of occupation number of the spin states $\epsilon_{\Uparrow}$ and $\epsilon_{\Downarrow}$ (not shown). These occupation numbers are calculated as usual by integrating the imaginary part for the corresponding matrix element of the lesser Green functions $G_{\Uparrow, \Downarrow}^{<}$. These functions are given by the Keldysh equation $G_{\Uparrow}^{<}=\left(G^{R} \Sigma^{<} G^{a}\right)$. The dips and peaks in the spin currents are rather well resolved due to the selected value of the spin splitting $\Delta$. By decreasing $\Delta$ the peaks and dips merge. These results show that when electrons tunnel through the side-coupled $\mathrm{QD}$, the spin interference is strongly affected. In fact, the filtering of spins with different polarizations in the output leads decreases considerably and therefore the splitter efficiency drops from $65 \%$ to $25 \%$.

In Fig. $2 \mathrm{c}$ we present the charge currents (e.g. $J_{\beta}=J_{\beta, \uparrow}+J_{\beta, \downarrow}$ ) in the output leads. As expected, $J_{\beta}$ and $J_{\gamma}$ exhibit Fano lineshapes. It is well known that a Fano lineshape is analytically described by the function $\frac{(E+q)^{2}}{E^{2}+1}$, where $E$ is the energy and $q$ is the so-called Fano parameter describing the asymmetry of the line. In the mesoscopic Fano regime the energy $E$ depends also on the gate potential which sets the QD on resonance (see e.g. [13]). In Fig. $2 \mathrm{c}$ one observes that the Fano parameters have different signs $\left(q>0\right.$ for $J_{\beta}$ and $q<0$ for $\left.J_{\gamma}\right)$. This means for example that as the QD levels sweep the bias window, the interference at contact $\beta$ is firstly suppressed and then slightly enhanced. The opposite signs of the Fano parameter are easily understood if we analyze the behavior of spin currents given in Fig. 2b. The Fano lineshape of $J_{\beta}$ starts with a dip because $J_{\beta, \uparrow}$ drops suddenly whereas $J_{\beta, \downarrow}$ is still constant as $\epsilon_{\Downarrow}$ is not yet within the bias window. On the contrary, $J_{\gamma}$ develops a Fano peak first because $J_{\gamma, \uparrow}$ increases at resonance.

The signs of the Fano parameters are reversed if one changes the sign of the magnetic flux. In this case $J_{\beta, \uparrow}$ displays a peak followed by a dip (not shown). This is due to the fact that the Fano parameter is a periodic function of the magnetic flux $\Phi$. While both $J_{\beta}$ and $J_{\gamma}$ display clear Fano lineshapes, the input current $J_{\alpha}$ shows two small dips and its amplitude does not decrease considerably on resonance (not shown). This is quite different from the two lead case where both currents exhibit similar Fano resonances. Note that in the three-lead geometry current conservation imposes $J_{\alpha}=J_{\beta}+J_{\gamma}$ which leaves room for different shapes of the currents.

The opposite behavior of the charge currents allows one to simultaneously control the amplitude of the two output currents by tuning the gate potential applied on the QD. As seen in Fig. 2c
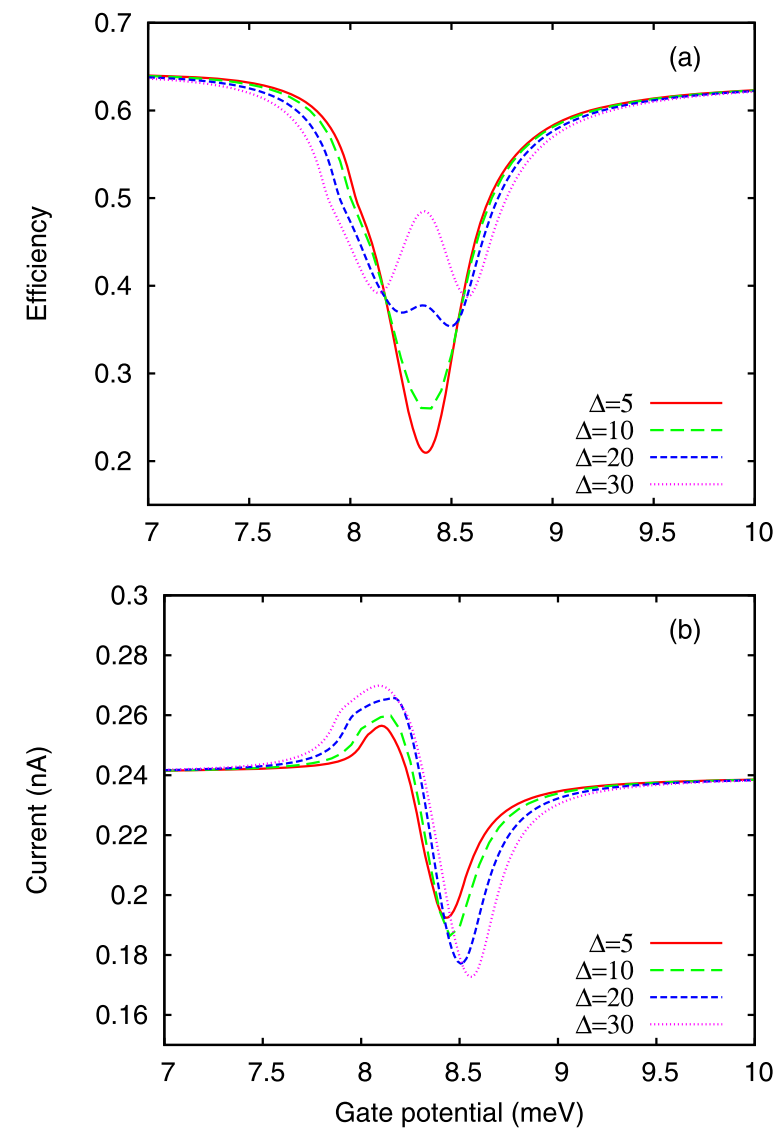

Fig. 3. (Color online.) (a) The splitter efficiency $E_{\uparrow, \downarrow}$ as a function of the gate potential $V_{g}$ for different values of the spin splitting between the QD levels (the parameter $\Delta$ is defined in the text). As $\Delta$ increases $E_{\uparrow, \downarrow}$ develops a maximum and two minima. (b) The charge current $J_{\gamma}$ as a function of $V_{g}$ and $\Delta$. Other parameters are $\alpha_{R}=0.27 \times 10^{-11} \mathrm{eVm}, \Phi=0.99 \Phi_{0}, \tau_{r}=0.001$.

at $V_{g} \sim 8 \mathrm{meV}, J_{\gamma}$ is almost twice larger than $J_{\beta}$ while if $V_{g}$ is changed to $V_{g} \sim 8.4 \mathrm{meV}$ the situation is reversed. This fact is made possible by the interference processes in three-lead ring and by the Fano effect.

The efficiency of the splitter is strongly affected when electrons pass through the side-coupled QD because this additional tunneling brings in a different phase of the electron's wavefunction, altering therefore the spin interference. Moreover, one expects to see a dependence on the spin splitting $\Delta$ as well. Indeed, Fig. 3a confirms that the efficiency $E_{\uparrow, \downarrow}$ decreases at resonance and that in general splitter regime is no longer possible in the presence of the side-coupled dot. Interestingly, the dependence of $E_{\uparrow, \downarrow}$ on $\Delta$ is not monotonous. Fig. 3a shows that for $\Delta=5$ the efficiency drops by almost $45 \%$ while for $\Delta=20$ one notices the appearance of two dips and of a local maximum; these dips are clearly established for $\Delta=30$. In order to understand the emergence of the additional peak at larger $\Delta$ it suffices to observe that a large spin splitting of the QD levels diminishes the overlap of the peaks and dips for the spin-up and spin-down currents. This means that at certain values of the gate potential (e.g. $V_{g} \sim 8.25 \mathrm{meV}$ in Fig. $2 \mathrm{~b}$ ) one still has a good spin filtering and therefore a moderate splitter efficiency $(\sim 48 \%)$. On the other hand, the severe drop of $E_{\uparrow, \downarrow}$ at small spin splittings follows from the fact that the $J_{\beta, \uparrow}$ and $J_{\gamma, \downarrow}$ are almost simultaneously suppressed so the numerator in Eq. (11) decreases.

The effect of the spin splitting within the side-coupled QD on the Fano lineshapes is presented in Fig. 3b. It is clear that the distance between the Fano peaks and dips increases with $\Delta$ as well 

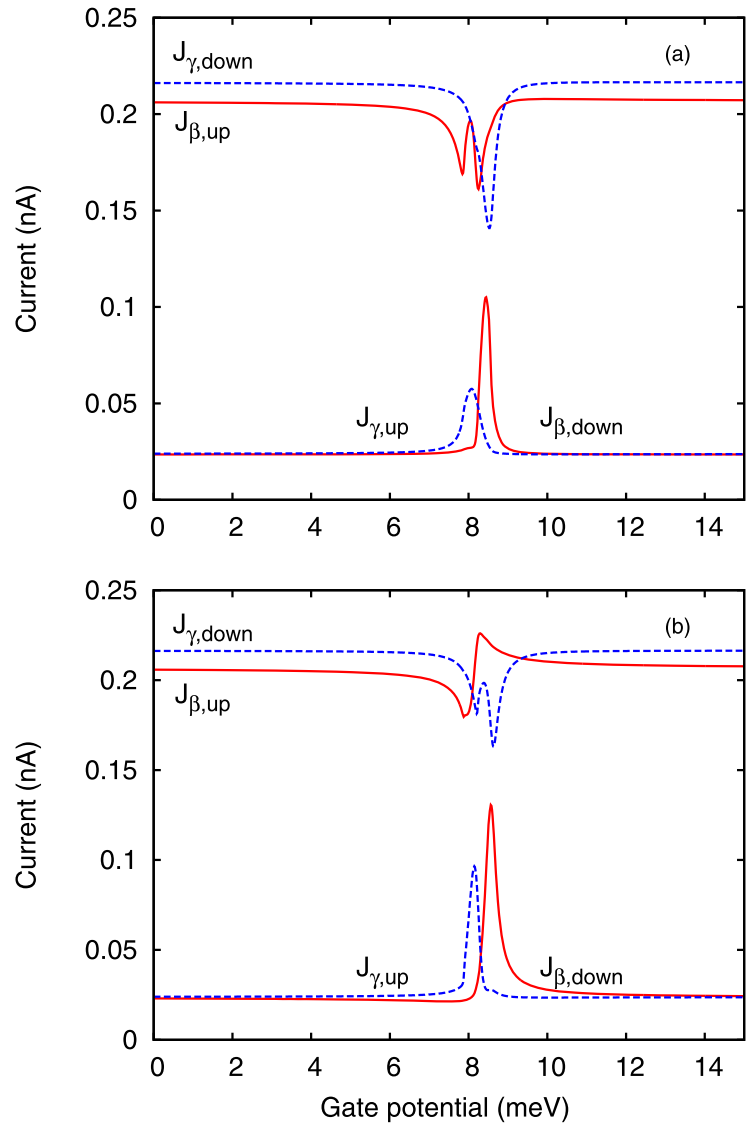

Fig. 4. (Color online.) The spin currents in the output leads as functions of the gate potential $V_{g}$ at two different locations of the side-coupled quantum dot. (a) $\theta_{p_{0}}=$ $3 \pi / 8$ and (b) $\theta_{p_{0}}=\pi / 4$. The discussion is made in the text. Other parameters are $\alpha_{R}=0.27 \times 10^{-11} \mathrm{eVm}, \Phi=0.99 \Phi_{0}, \tau_{r}=0.001$.

as the amplitude and width of the Fano lineshapes. A similar behavior is observed for $J_{\beta}$ (not shown).

The Fano interference in the system considered here is much more complicated than in the case of a spinless ring with an embedded $\mathrm{QD}$. In that case the interference involves roughly a background contribution given by electrons traveling on the bare arm of the ring and many resonant contributions associated with electrons passing at least once through the QD. For the ring with the side-coupled dot one should have in mind that: (i) the states corresponding to the bare ring levels are spinors; (ii) each such spinor has in general non-vanishing up and down components with respect to the $z$-axis; (iii) the amplitudes of these components depend on the site $p$ where we evaluate the spinors $\left|\psi_{l s}\right\rangle^{1}$ so one expects the tunneling to and from the dot to depend on the location of the contact site $p_{0}$ on the ring. Moreover, each of the spinors associated with the four levels within the bias window in Fig. 2a is involved in the interference.

In order to reveal the complex interplay between the Fano and Rashba interferences, we performed simulations for different locations of the contact site $p_{0}$. In Figs. $4 \mathrm{a}$ and $4 \mathrm{~b}$ we present the four spin currents for $p_{0}=16$ (i.e. $\theta_{p_{0}}=3 \pi / 8$ ) and for $p_{0}=11$ (i.e. $\left.\theta_{p_{0}}=\pi / 4\right)$. The location of the leads remains the same. We shall discuss the behavior of spin currents only, the reader can guess the charge current features by adding the spin currents in each output lead. When comparing these plots with the results shown in Fig. 2b one notices that the spin currents are substantially mod-

\footnotetext{
1 This can be easily seen from the definitions the spinors in Eqs. (5), (6) and by having in mind that $\left|\phi_{l}\right\rangle=\frac{1}{\sqrt{N}} \sum_{p=1}^{N} e^{i l \varphi_{p}}|p\rangle$.
}

ified when $p_{0}$ is changed. Fig. $4 \mathrm{~b}$ reveals that instead of a single pronounced dip, $J_{\beta, \uparrow}$ develops two smaller dips. Also, $J_{\beta, \downarrow}$ peaks from $0.0025 \mathrm{nA}$ to $0.1 \mathrm{nA}$. The currents in the lead $\gamma$ behave similar to the ones in Fig. $2 \mathrm{~b}$. When $p_{0}=11$ a Fano line with small amplitude is obtained for $J_{\beta, \uparrow}$ whereas $J_{\beta, \downarrow}$ shows two dips. The filtered currents $J_{\beta, \downarrow}$ and $J_{\alpha, \uparrow}$ display simple but slightly asymmetric peaks.

It is clear that the configuration $p_{0}=21$ (i.e. $\theta_{p_{0}}=\pi / 2$ ) leads to the simplest behavior of the spin currents (i.e. simple peaks or dips). Note that the number of peaks and dips is not the same for the spin currents in different leads (for example in Fig. 4a $J_{\beta, \uparrow}$ develops two dips while $J_{\beta, \downarrow}$ has only one). This happens because the output currents result from the spin interference and the Rashba spin-up and spin-down phases acquired by the electrons traveling on different paths are not equal.

We also performed numerical simulations for rings of different radii and found similar results, provided the parameters for the spin-splitter regime are appropriately tuned.

\section{Conclusions}

We studied the spin interference and the mesoscopic Fano effect in a Rashba ring coupled to three leads and a side-coupled quantum dot. The latter is controlled by an applied gate potential. We used the non-equilibrium Green's function formalism to calculate the spin and charge currents in the output leads as a function of the gate potential applied on the dot. As long as the tunneling through the QD is forbidden the ring acts as a spin splitter provided one suitably adjusts the constant perpendicular magnetic field, the strength of the Rashba spin-orbit coupling and the bias applied across the leads. We find that when a pair of spin-split levels of the QD enter the bias window the charge currents display Fano lineshapes associated with resonant tunneling to and from the quantum dot.

The two lineshapes have Fano parameters with different signs, which leads to a simultaneous increase (decrease) of the currents in the first (second) output lead. These features are explained by the spin interference along the ring in the presence of the sidecoupled dot. The effect of the intradot spin splitting on the splitter efficiency and on the Fano lineshapes is also analyzed. It turns out that the interference between the clockwise and counterclockwise spin states is strongly affected by the presence of the QD, the spin currents depending as well on the site where the QD is attached.

This system can be easily realized in experiments and offers a way to control the charge currents in different output leads by tuning a single parameter on the QD, namely the gate potential.

\section{Acknowledgements}

V.M. and M.T. acknowledge the financial support from PNCDI2 program under grant No. 515/2009, and the hospitality of the Bilkent University where this work was initiated. B.T. acknowledges support from TUBITAK and TUBA.

\section{References}

[1] J. Splettstoesser, M. Governale, U. Zülicke, Phys. Rev. B 68 (2003) 165341.

[2] D. Frustaglia, K. Richter, Phys. Rev. B 69 (2004) 235310.

[3] P. Földi, O. Kálmán, M.G. Benedict, F.M. Peeters, Phys. Rev. B 73 (2006) 155325

[4] S. Souma, B.K. Nikolić, Phys. Rev. B 70 (2004) 195346.

[5] J. Nitta, F.E. Meijer, H. Takayanagi, Appl. Phys. Lett. 75 (1999) 695.

[6] T. Bergsten, T. Kobayashi, Y. Sekine, J. Nitta, Phys. Rev. Lett. 97 (2006) 196803.

[7] P. Földi, O. Kálmán, M.G. Benedict, F.M. Peeters, Phys. Rev. B 73 (2006) 155325

[8] R. Citro, F. Romeo, M. Marinaro, Phys. Rev. B 74 (2006) 115329.

[9] S. Bellucci, P. Onorato, J. Phys.: Condens. Matter 19 (2007) 395020.

[10] G. Cohen, O. Hod, E. Rabani, Phys. Rev. B 76 (2007) 235120.

[11] V. Moldoveanu, B. Tanatar, Phys. Rev. B 81 (2010) 035326

[12] V. Moldoveanu, B. Tanatar, Phys. Lett. A 375 (2010) 187. 
[13] K. Kobayashi, H. Aikawa, S. Katsumoto, Y. Iye, Phys. Rev. Lett. 88 (2002) 256806.

[14] S. Katsumoto, J. Phys.: Condens. Matter 19 (2007) 233201.

[15] A.E. Miroshnichenko, S. Flach, Y.S. Kivshar, Rev. Mod. Phys. 82 (2010) 2257.

[16] A. Fuhrer, P. Brusheim, T. Ihn, M. Sigrist, K. Ensslin, W. Wegscheider, M. Bichler Phys. Rev. B 73 (2006) 205326.
[17] F. Chi, J.-L. Liu, L.-L. Sun, J. Appl. Phys. 101 (2007) 093704.

[18] W. Gong, Hui Li, Y. Han, G. Wei, Phys. Lett. A 375 (2011) 1333.

[19] D.N. Son, N. Arboleda, W.A. Dino, H. Kasai, Eur. Phys. J. B 57 (2007) 27.

[20] F.E. Meijer, A.F. Morpurgo, T.M. Klapwijk, Phys. Rev. B 66 (2002) 033107.

[21] B. Berche, C. Chatelain, E. Medina, Eur. J. Phys. 31 (2010) 1267. 